\title{
Study on the Visual Expression of Dunhuang Frescoes and Zhongyuan Tomb Murals in the Tang Dynasty
}

\author{
Zhaoxia Li \\ Xi'an Polytechnic University \\ Xi'an, China
}

\begin{abstract}
In the Tang Dynasty, Dunhuang and Zhongyuan both entered the prosperous period of fresco creation, and many Western painters came to Dunhuang and Zhongyuan to draw murals, which brought inevitable collision and blending with the original Zhongyuan native painting. However, due to the comprehensive factors of geographical position, cultural background and cultural inertia and so on, Dunhuang and Zhongyuan show their own different unique style of murals in the drawing methods and expressive results. This paper makes comparison and judgment of the related literature and the existing murals, attempts to sort out the similarities and differences of the Dunhuang frescoes and the Zhongyuan tomb murals in the Tang Dynasty in the artistic styles of line, color and shape after this blending, looking forward to giving some revelation and reference for the creation and development of Chinese modern heavy-colored paintings.
\end{abstract}

Keywords-tomb murals in the Tang Dynasty; Dunhuang fresco; artistic style

\section{THE EXPRESSION OF DIFFERENCES BETWEEN DUNHUANG AND ZHONGYUAN MURALS IN THE TANG DYNASTY}

As the starting point of the ancient Silk Road, Chang'an had opening politics and prosperous economy in the Tang Dynasty, keeping frequent business contacts with the Western Regions, Central Asia and European countries, and at this time, mural creation also entered its prosperous period. While in Zhongyuna, the palaces, governmental offices and temples most were decorated with murals in the subject matter of religion, historical stories, real character and so on.

At the same period, there were many merchants and religious propagators frequently going through Dunhuang because Dunhuang is the traffic artery of the ancient Silk Road access to the Western Regions. From the Han and Wei Dynasties, India Buddhism began to spread along the Silk Road, and through a long history, the world-famous Dunhuang mural art is created, with the theme of Buddhism continued.

(1) According to the Dunhuang literature, it is known that "in The Tang Dynasty and the Five Dynasties, Dunhuang lived Han Chinese, Tibetans, Sogdians, Tuyuhuns, Enhens, Uighurs, Nanshans, and a small number of Persians, Indians and etc. some of them preached Buddhism or other religions, spread the Western history and scientific and cultural knowledge to China, and some engaged in the commercial economy or acted as envoy rushing about in the ancient way of exchanges between China and the West." [1] As the distribution center and transfer station of religious, cultural and material exchange, Dunhuang has the almost absolute historical inevitability for the collision and blending in culture and art. As the Indian Buddhist art of Gandhara style is regarded, under the influence of realistic style of the Greek art that expresses the object image by use of light and shadow, the Hellenistic Indian artistic style is spread east to Dunhuang in the process of Buddhism's development towards the east, and after blending with the local style and Zhongyuan style, the Dunhuang art with regional characteristics formed. Through the Silk Road, this Western painting style also influenced the Zhongyuan painting style.

(2) In the Tang Dynasty, Zhongyuan's Buddhist art also formed in a stable style, and at that time, the painters all are good at Buddhist painting. According to records, Zhou Fang created the image of "Water-Moon Guanyin" and the new style, known as "Zhou's Style"; Wu Daozi, good at the painting of Buddhist figures, created the new style of religious painting, known as" Wu's Style". Chang'an is an international metropolis and also the starting point of the Silk Road, so it is filled with various people and things from the Western Regions and Rome. These enriched Zhongyuan's original cultural tradition but did not change it completely, because Zhongyuan had its ineradicable culture of thousands of years, and it absorbed, contained and accepted foreign culture. "Zhongyuan's native painting always attached importance to the artistic conception and the forcefulness of lines, it will not give up the tradition to absolutingly accept the Western style of painting that takes the form and structure, three-dimensional sense and color as the primary standard. [2] So even if Zhang Sengyao studied Indian concave and convex dyeing method and Weichi Yiseng's painting style was famous in the whole city of Chang'an in the Tang Dynasty, they only have a subtle impact on Zhongyuan painting in the entire art history, and will not become the mainstream style. "The Western style has been integrated into Chinese traditional techniques with a subtle influence." [3]

(3) During the hundreds of years after Western Buddhism is introduced into Dunhuang, it had been fully integrated with 
the native arts in the Tang Dynasty, and had a profound impact on the mural art in Zhongyuan. The process of its artistic evolution is also deeply marked with the religious communication, cultural customs and commercial prosperity.

It is recorded in the Subsequent Record on Paintings: "Yuchi Yiseng is good at painting foreign ghosts and gods with strange shapes and looks, rarely followed by Chinese painters ... although his stroke method is different from that of Chinese painting, his painting is full of righteousness and dignity" [4]. It is said in the Record on Famous Paintings of Past Dynasties: "the monk Jingang Sanzang, from Ceylon, is good at painting the image of Western Buddha, with smooth, skilled and steady drawing techniques" [5]. These records indicate that the Western style and the Zhongyuan style along the Silk Road are influenced by each other but not replaced by each other, and both of them have their own artistic characteristics.

\section{THE LINE APPLICATION FEATURES OF DUNHUANG FRESCOES AND ZHONGYUAN TOMB MURALS IN THE TANG DYNASTY}

It is said in the Record on Famous Paintings of Past Dynasties: "for the character paintings, the most important is to make the figure is alike, for which the bearing must be expressed, while it ultimately depends on the stroke technique".[6] so it is visible that line is important in Chinese painting. In the Tang Dynasty, the tomb murals in Zhongyuan and the Dunhuang Grotto murals at the traffic link of the Silk Road have their different emphasis in applying painting lines.

(1) In the Tang Dynasty, Zhongyuan tomb murals adopted line modeling, and the steady and strong iron-wire stroke played a leading role in the picture. Line is the main aesthetic factor in the picture, color and other painting factors are attached to the existence of lines, and if the line is discarded, the picture will no longer exist. The lines are arranged properly according to the structure, with appropriate density; the lines are round and smooth, uniform and even, with subtle change in thickness. The entire clothes lines are concise, clear and inclusive, belonging to the category of sparse style painting. Strokes are steady and powerful, comfortable and smooth, concerted freely, stressing on the application of brush and ink, which are typical iron-wire strokes. Wu Daozi is the famous painter in Zhongyuan in the Tang Dynasty, it is recorded in the Record on Famous Paintings of Past Dynasties: "Zhang Sengyao and $\mathrm{Wu}$ Daozi have excellent painting techniques, easily drawing the image..."; "In the painting of landscape, we should induce more with less, fan out from point to area, and not make excessively full and dense, so you will not worry about little painted only when it includes the key" [7] This will also become a popular painting concept, which does not take particular and fine depiction as standard, and it certainly will affect the aesthetic consciousness at that time and become a popular style of painting. The tomb murals of the Tang Dynasty have the marked features of popular "coke-link lightcolor style" in Zhongyuan (the Central Plains) and of "Wu's Style" sparse painting[8].

(2) In the murals of Dunhuang in the Tang Dynasty, the lines also played an important role in the paintings, but it has a clear difference of line expression way from that of Zhongyuan tomb murals in the Tang Dynasty. The Dunhuang fresco in the Tang Dynasty more continues the block and area modeling way in the Western painting, and the lines mainly have the role of defining the image in the picture. It is wholly characterized by fine, smooth and beautiful lines, like the flying ribbons, intertwined with a variety of lines such as thin and long clothing lines, and fine decorations, showing its richness and delicacy. This specific performance is achieved by the following process: first take earthy red as shaping line before painting, then use color block to express the form and structure, and then draw ink lines, color lines, white lines of different depth and thickness according to the needs, and even some parts do not need lines. These lines should be flexibly changed according to the picture needs, making free and comfortable. Looked far, the picture seems a full combination of color blocks, not only stressing on the role of lines and the aesthetic taste for lines, and through the integration of color and line, they jointly create the picture. Additionally, it should be noted specially about Weichi Yiseng's line expression (who is a painter of Yutian in the Western Region). According to records, Weichi Yiseng, the painter of Yutian has "a compact and powerful stroke, like curving and twisting iron wire, and the lines are even and fine, forceful and elastic. As Weichi's royal family in Yutian, Weichi Yiseng was recommended by the king of Yutian to the imperial court of the Tang Dynasty in Zhongyuan (the Central Plains) and praised by the people as "all wonderful" at that time because of his "wonderful painting", so his painting style should also be the important composition or representative of the artistic style of the paintings popular in the Western Region; and the Dunhuang murals in the Tang Dynasty continue this style of Western painting to a large extent.

Through the comparison, it can be seen that in the application of lines, Tang dynasty tomb murals make the frame with ink line and manage the color with ink line, while Dunhuang fresco takes more emphasis on the integration of color and line, creating the picture with both line and color.

\section{THE REGIONAL DIFFERENCES IN COLOR APPLYING METHOD OF DUNHUANG FRESCOES AND ZHONGYUAN TOMB MURALS IN THE TANG DYNASTY}

The Dunhuang frescoes and the Zhongyuan tomb murals in the Tang Dynasty both are traditional Chinese heavy-colored realistic painting, both mainly take mineral color as pigments and use the principle of structural color, but they have their own characteristics in color setting. Their color setting method mainly includes two kinds, of which one is the traditional flat spreading method with lines in Zhongyuan (the Central Plains) and the other is the concave-convex blooming method of the Western paintings. The color setting has difference in thickness, including the thin dyeing of "color applying style" and the thick dyeing of "color overlapping style". [9]

(1) For Tang dynasty tomb murals, the color is mainly used to support the role of lines, and the dyeing combines the flat spreading technique and blooming technique. Facial blooming color is pink or ember spreading outward from the cheeks, which is high dyeing method, with accurate blooming parts, symmetrical scumble and adept skills. For clothes, some adopt 
flat spreading method of the Central Plains, and more adopt sfumato with high dyeing and low dyeing combined according to the concave-convex structure of texture. Strong color is used at the significantly concave part along the lines of clothing texture, making a gradual transition to another line; or high dyeing method is used at the significantly convex parts between two clothing texture lines, redyeing bulge with transition from the convex part to the lines at both sides, with strong sense of concavity and convexity. Pay special attention to the stroke when setting the color, in accordance with the direction of clothing texture, with a close combination between line and color. First set line, and then color in the scope framed by the line, without color covering or hiding the line. Apply color thinly and lightly, not deliberately requiring full and complete details. The color has a rich change in degree, with the effect of ink painting in the Song and Yuan Dynasties, full of ink fragrance. The color of the fresco is mainly vermeil, crimson, chocolate, rouge, medium yellow and other warm colors, with peaceful, warm and free hue, graceful and quiet style.

Tang Dynasty tomb murals generally adopt the "color applying style" painting of thinner dyeing, which is the emergence of "Wu's style" for "light coloring". India's sfumato to show motion sensing had been spread to the Central Plains from the Western Regions in the Han and Wei dynasties. It is recorded in the History Records of Jiankang that Zhang Sengyao painted concave-convex flowers in a temple, having a scumble from low to high, to highlight its concave-convex sense, which was India's blooming method. In the Tang Dynasty, this blooming method has been transformed into a basic technique of Chinese painting.

(2) Color is the primary factor in the painting of Dunhuang murals, and it can define the image through the contrast of color block. Most of the Dunhuang murals in the Tang Dynasty apply the Western painting method and pay attention to the color-overlapping style of concave-convex stereography, making repeated color blooming from low to high. For the face, it also mainly adopts the low dyeing in the Western region, but not as crude as that at the early stage of introduction of Buddhism, which is completely different from the facial performance of that in Zhongyuan tomb murals. The whole mural uses color blooming, massively coloring, and the picture has a three-dimensional sense. On the white or khaki background, stone blue and stone green are used mainly, with minium, sienna, ocher, ocher yellow, brown, white and black interluded, to present a peaceful, quiet and gentle atmosphere of a Buddha state, full of strong exoticism. The facial painting of providers is different from that of Buddha and Bodhisattva, but it is a very pattern style of painting, not changing the pattern according to the clothing texture or showing a volume sense; it has flat, color-block, and strong decorative sense. The sfumato of high dyeing is used for facial part, similar with Zhou Fang's Dao Lian Tu, having obvious traces of the Central Plains painting, which should be the appearance of Zhongyuan popular fine style of painting in the Dunhuang murals. It is recorded in the Yang Shengan Volumes: "Yuchi Yiseng is good at painting concave-convex flower"; it is said in the Qinghe Calligraphy and Painting House: "Yiseng is good at coloring ... works is wondrously fine and beautiful." These indicate that concave-convex blooming method is popular in the Western Regions at that time.

Through the comparison, we can see that Dunhuang frescoes and Zhongyuan tomb mural in the Tang Dynasty both are from the combination of Western painting and the Central Plains painting, and they have emphasis in different points and aspects after the combination.

\section{THE MODELING DifFERENCES BETWEEN THE DUNHUANG FRESCOES AND ZHONGYUAN TOMB MURALS IN THE TANG DYNASTY}

\section{A. Dunhuang Frescoes Have More Character Models That Show The Impact of Western Painting on It.}

The characters have a proper body structure proportion, symmetric body, full and round limbs, natural and reasonable joint connection. At the same time, they also have fine details, such as various and beautiful hand gesture, reasonable and comfortable proportion, delicate and accurate knuckles. This is clearly influenced by the Greek art, the source of Buddhist painting. "Bodhisattva's upper body half-naked or Sengqizhi, jewelry crown, gem necklaces and jade bracelets, long shawl, wiggly posture, bare foot" [10], all these are not dressing of people in the Central Plains, but retain the pattern of Greece, India and Persia, while the image modeling and inherent gaseity of the characters has completely Chineseized.

The Buddhist images in the Dunhuang frescoes show the social fashion in the Tang Dynasty. The image of face and hand, inherent gaseity and other aspects all reveal the China's Buddhist art image. The transcendent Buddha, forgiving and compassionate Bodhisattva, graceful and charming dancers, valiant and martial Hercules, free flying Apsaras and aflutter flowers together create a positive, happy, friendly and warm religious atmosphere, making people not help longing for and aspiring after it. Art is a portrayal of real life, and the Dunhuang frescoes belong to the Buddhist world but this art atmosphere appeared only in the Tang Dynasty where people had political freedom, economic prosperity, cultural tolerance, rich life. The Dunhuang frescoes in the Tang Dynasty are the perfect fruits of the Western style painting development in the social environment of China in the Tang Dynasty.

\section{B. Zhongyuan Tomb Murals in the Tang Dynasty Have Different Modeling Types from the Dunhuang Frescoes in the Same Period.}

The performance of body structure continues the traditional way of the Central Plains to describe the appearance through spirit. The painting of characters has changed the former proportion of large head and small body, tending to coordination. Maids have mellow and pretty face, and some of them who are slim show a three-up-and-down body in a quiet and mellow state. These murals pay attention to the mind description of various characters that watch bird or catch cicadas, hold cups or fans, play dance or music, with different ages, different gestures and different minds, portrayed vividly. Soldiers stand at attention, with a handsome image; envoys are portrayed accurately, with prominent features and enough expression, which has reached the level of portraits. The 
characters' dress, finery, look, utensils, activities, etc., all are the direct presentation of the real life. The characters wear shawl and robe, whose body shape under clothes is not deliberately depicted, specially the gesture of hand is also described generally, which shows a relatively large difference from that of Dunhuang frescoes. Tang dynasty tomb murals have no peace and happiness of Buddha world, but provide a quiet and cozy state of noble life.

For modeling, Dunhuang frescoes in the Tang Dynasty become the model among the paintings that describe spirit through body shape, reflecting the expression of the characters on the basis of accurate body structure. While the characters in the Tang Dynasty tomb murals are painted with the spiritbased description of body shape as the aesthetic standard. On the basis of enough expression, some details of the body shape can be abandoned, just as said in Zhang Yanyuan's Record on Famous Paintings of Past Dynasties: "painting according to the artistic conception must contain its body shape". [11] It can be said that this distinction is a different form of art produced under the guidance of different painting concepts.

\section{CONCLUSION}

A. The Dunhuang Frescoes and the Zhongyuan Tomb Murals in the Tang Dynasty Have Their Blending and TheirOwn Emphasis on Shape, Line, Color and Painting Concept.

In the Tang Dynasty, the Western painting has subtly influenced Zhongyuan paintings through the introduction of Buddhism into the Central Plains for centuries, and the new style of Zhongyuan painting has formed, wholly showing a painting style of "bold and fine strokes, graceful and elegant color" [12]. Dunhuang frescoes completed the perfect combination of the Western style and the Zhongyuan style, to reveal the gaseity and spirit of Zhongyuan people in the Tang Dynasty under the Western style of painting. In the Tang Dynasty, Dunhuang grotto murals in the remote place have differences in performance technique from the tomb murals in the Central Plains, but they make a reference and integration from each other.

\section{B. The Dunhuang Frescoes and Zhongyuan Tomb Murals in the Tang Dynasty Show A Similar Aesthetic Taste Due to Their Background in the Same Period.}

The tomb murals in the Tang dynasty use thick, heavy and strong iron-wire strokes, but they don't make us feel stiff or repressive because of concise and smooth clothing lines, thin and light color, quiet and comfortable character models, which always show the comfortable and cozy life of nobles in the Tang Dynasty. Dunhuang fresco in the Tang Dynasty have free and easy lines, brilliant and splendid color, cheerful and demure character modeling, everywhere showing the happiness and beauty of Buddha world. Generally, the frescoes of the Tang Dynasty give a relaxed and joyful, cheerful and free art atmosphere, which directly reflect the open and free mental outlook in the Tang Dynasty.

\section{It Has Significant Enlightenment and Guidenance to Study the Similarities and Differences between the Dunhuang Frescoes and the Zhongyuan Tomb Murals in the Tang Dynasty.}

After comparison, we can conclude that on the one hand, so high achievements of mural art in the Tang Dynasty have a very important relationship with the open and wide mind of the Tang Dynasty to accept the Western painting techniques. The Western style of painting is integrated with our national painting style to form a new painting style, bringing fresh vitality for Chinese painting, enriching the form of Chinese painting and creating a new tradition. So we should treat the foreign style with an open mind in the artistic creation, absorbing the good and abandoning the bad, then we will drive the development and innovation of our national painting.

On the other hand, the colorful paintings in the Tang Dynasty gradually fade out of the stage of history after the Song and Yuan Dynasties and are replaced by ink paintings. Fortunately, in the eastward process of Buddhism, this painting style is spread to China's neighbor Japan and preserved partly. After the Meiji Restoration, with the development of Japanese painting, this painting is developed in materials and application techniques and other aspects to a certain extent; in the early 1990 s, a group of people with lofty ideals went to learn, so that the tradition of murals in China was picked up and developed. We should open our mind to accept the back of the mural tradition; I believe the colorful Chinese paintings must have a new development in the new era through the painting artist's unremitting efforts on the basis of our profound traditions, rich murals remnants and the development of Japanese painting.

The frescoes and murals respectively in Zhongyuan and in Dunhuang in the Tang Dynasty both are the outcome from the integration of painting styles of the Western Regions and of the Central Plains, but due to their different cultural background and geographical location, they both reflect some integrated but differentiated points. The Dunhuang frescoes and the Zhongyuan tomb murals in the Tang Dynasty have their own emphasis on the shape, lines, color and others, but because of the same dynasty they are in, they present similar aesthetic taste with each other.

\section{REFERENCES}

[1] Zheng Binglin. The Manuscripts and Physiognomical Theory of Dunhuang and Providers' Portrait in Dunhuang Grottoes [M]. Gansu: Dunhuang Journal 2006 (4): p2

[2] Zhou Jiyin. The Theory on Chinese Paintings in the Past Dynaties [M]. Jiangsu Fine Arts Publishing House, 2007: 915

[3] Zhao Feng. On Art Archeology of the Silk Road [M]. Cultural Relics Press, 2007 (3): P.240

[4] Li Qing. On Kroraina Shanshan Art [M]. Beijing: China Bookstore. 2005.

[5] Yan Zong. Subsequent Reconrd on paintings - On the Paintings in the Tang Dynasty and the Five Dynasties [M]. Changsha: Hunan Fine Arts Publishing House, 1999: 3

[6] Zhang Yanyuan. Famous Paintings in the Past Dynasties - Tang [M]. Hangzhou: Zhejiang People Art Publishing House, 2011: 151.

[7] Same with [6] 28.

[8] Same with [6] 16.

[9] Tang Changdong. Stroke And Paintings in the great Tang Dynasty [M]. Xi'an: Shaanxi Tourism Press, 1999 (8): P6. 
[10] Niu Kecheng. Colorful Chinese Paintings [M]. Changsha: Hunan Fine Arts Publishing House, 2005 (5): P57-59.

[11] Duan Wenjie. China Dunhuang Frescose ·Tang [M]. Tianjin: Tianjin People's Fine Arts Publishing House, 2010 (6): P10-15.

[12] Same with [6] P16

[13] Li Xingming. Study on Tang Dynasty Tomb Murals [M]. Xi'an: Shaanxi People's Fine Arts Publishing House, 2005 (10): P294.. 